\title{
Towards Semi-Automated Satellite Mapping for Humanitarian Situational Awareness
}

\author{
Stefan Voigt, Elisabeth Schoepfer, Christoff Fourie and Alexander Mager \\ German Aerospace Center (DLR) \\ German Remote Sensing Data Center (DFD) \\ Oberpfaffenhofen, Germany
}

stefan.voigt@dlr.de; elisabeth.schoepfer@dlr.de; christoffel.fourie@dlr.de; alexander.mager@dlr.de

\begin{abstract}
-
Very high resolution satellite imagery used to be a rare commodity, with infrequent satellite pass-over times over a specific area-of-interest obviating many useful applications. Today, more and more such satellite systems are available, with visual analysis and interpretation of imagery still important to derive relevant features and changes from satellite data. In order to allow efficient, robust and routine image analysis for humanitarian purposes, semi-automated feature extraction is of increasing importance for operational emergency mapping tasks. In the frame of the European Earth Observation program COPERNICUS and related research activities under the European Union's Seventh Framework Program, substantial scientific developments and mapping services are dedicated to satellite based humanitarian mapping and monitoring. In this paper, recent results in methodological research and development of routine services in satellite mapping for humanitarian situational awareness are reviewed and discussed. Ethical aspects of sensitivity and security of humanitarian mapping are deliberated. Furthermore methods for monitoring and analysis of refugee/internally displaced persons camps in humanitarian settings are assessed. Advantages and limitations of object-based image analysis, sample supervised segmentation and feature extraction are presented and discussed.
\end{abstract}

Keywords - satellite mapping, monitoring, humanitarian situational awareness

\section{INTRODUCTION}

During dynamic humanitarian operations, accurate and upto-date situational awareness is a key requisite for the management of response and supply capacities. Along with standard information and communication technology, the use of recent satellite imaging and derived map products by actors and decision makers are becoming more common. The spatial resolution and accessibility of civilian and commercial optical satellite imagery have increased significantly in the past years. The US government-mandated maximum resolution of publically available optical imagery has recently increased to 0.25 meters per $\operatorname{pixel}^{1}$. In parallel, the number of commercial providers of sub-meter satellite data has increased globally. This allows for better access to almost daily satellite observation worldwide.

${ }^{1}$ Refer to http://www.reuters.com/article/2014/06/11/digitalglobe-imageryidUSL2N0OR2UX20140611 (accessed on August 11 $1^{\text {th }}, 2014$ )
Together with this increased availability and accessibility of satellite imagery, the demand for robust and easy to use analysis methods for refugee camp mapping, situational/environmental monitoring, damage assessment etc. has risen sharply. High resolution optical satellite imagery can sometimes, and to a good extent, be read and interpreted by nonexperts. Nevertheless, comprehensive, rapid, robust and reliable information extraction for optical sub-meter satellite imagery remains an important, challenging and demanding task.

With the wider use of satellite imagery higher expectations from the humanitarian sector can be observed: Faster turnaround times are expected when requesting an imaging/mapping activity. This includes programming the satellite, receiving the imagery, accessing, processing and extracting all relevant information and providing the derived material in form of maps, reports or in Geographic Information System (GIS)-ready format to the decision makers. Reactivity is not the only important factor, since robustness, reliability and completeness are key elements in operational rapid satellite mapping.

\section{CONSIDERATIONS ON SENSITIVITY AND SECURITY}

The level of detail in information, and the range of information, that can potentially be extracted from high spatial resolution satellite imagery for humanitarian mapping may be encompassing. It is suggested that during ongoing crisis situations great care should be taken when disseminating and publishing such derived information products, as to prevent any negative consequences to those intended to benefit from it. Most often, mapping and satellite-based monitoring in complex situations is generated with civilian and humanitarian intentions. However, if maps unintentionally depict military installations or other infrastructure details compromising one side of a conflict, this can result in a serious endangerment of the local population or relief personal.

Additionally, specific humanitarian mapping products, if endorsed by states and governments, can sometimes cause direct diplomatic concerns or implications when issued without sufficient caution. This can result in a conflict of interest. Mapping or monitoring information should be widely spread and shared in order to maximize beneficial use of the information. While the European Earth Observation program COPERNICUS uses "full and open access to information" as the basic principle in data policy, ethical and sensitivity con- 
cerns may inflict limitation to this general principle of free and open access. In certain situations, data or mapping results may better be held back from publication, at least temporarily, to avoid security issues and diplomatic friction.

A careful check regarding the sensitivity of satellite mapping products needs to be a routine element of humanitarian crisis mapping procedures. In the frame of such sensitivity considerations, the judgment of closely involved local actors as well as that of the international community (UN, NGOs) should be taken into account concisely. Satellite mapping and analysis results may and should be held back from the general publication in justified cases to prevent any adverse effects on an unfolding situation or to ensure the safety of all parties involved.

\section{STATE OF THE ART}

During the process of satellite data analysis and information extraction, visual analysis and interpretation of the imagery plays an important role. This is the case especially when deriving relevant geo-features and temporal changes relevant for assessing a humanitarian situation, e.g. in and around a refugee camp or when working in a conflict situation. While fully automated information extraction proved not to be reliable enough for humanitarian mapping, semi-automated information extraction methods and processing schemes, supported by welltrained image analysts, have been further advanced in the past years. The aim is to combine the situational and contextual perception as well as the cognitive skills of an experienced image analyst with computational power and the reliability of today's image processing environments.

Satellite based rapid mapping to support emergency and crisis management, has been developed and demonstrated for example by [1]. Globally, different satellite mapping programs have been set up with the majority of them supporting response to natural disaster and emergency management in general. In the frame of the European Union's Seventh Framework Program and the European Earth Observation program COPERNICUS (formerly known as GMES), substantial research and development activities are carried out to increase the scientific understanding as well as operational service capacities for humanitarian mapping and monitoring in particular. In the course of developing and operationalizing the Center for Satellite Based Crisis Information (ZKI) of the German Aerospace Center (DLR), the transition from mainly visual analysis and manual digitalization of geospatial features towards semi-automated object and feature recognition for operational humanitarian rapid mapping is of major importance. This also applies to the wider emergency mapping domain. Fig. 1 shows one of many examples where DLR/ZKI turned satellite data into useful information for humanitarian relief actors. In this case the German Federal Agency for Technical Relief (THW) was called to help UNHCR and other relief organizations with the building and expansion of the Al Zaatari Refugee Camp in Jordan. The camp provides shelter to thousands of refugees that have fled the civil war in Syria. In cases like these, different techniques for deriving the information from satellite imagery may be used. While visual analysis and manual digitization work can be tiresome and time consuming, automated procedures are

The research presented here received funding from the European Union's Seventh Framework Program for research; technological development and demonstration under grant agreement no 312912. often not reliable and stable enough to fully substitute the complex work of image analysts.

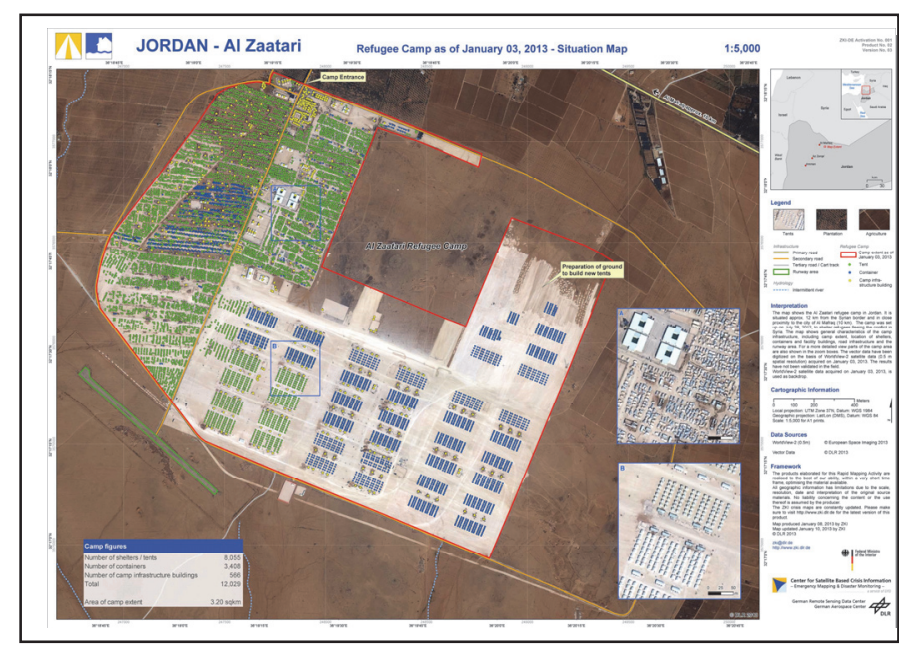

Fig. 1. Satellite based situation map generated by DLR/ZKI to support humanitarian operation of the German Federal Agency for Technical Relief (THW) at the Al Zaatari Refugee Camp (Jordan). The map was generated from WorldView-2 Satellite Imagery as of January 3, 2013. GIS layers with shelters and camp infrastructure were generated by visual interpretation and manual digitization (for more details and full resolution refer to www.zki.dlr.de).

Different processing schemes to partially automate the rapid mapping and information extraction processes are developed and applied at DLR/ZKI. They range from pixel-based supervised classification to object-based classification schemes and to dedicated and optimized sample/training supervised feature detection methods. A good overview on respective developments in automation of humanitarian mapping tasks in the context of the recent European Earth Observation activities is given by [2]. The authors also discuss the relevant aspects of mapping accuracy, benchmarking and validation of the respective mapping approaches.

\section{CURRENT TRENDS IN SATELLITE IMAGE ANALYSIS FOR HUMANITARIAN MAPPING}

\section{A. From visual interpretation to semi-automated feature extraction}

In the emergency mapping domain, which is mainly responding to natural disasters, work towards automation of image feature extraction, analysis and classification is widely conducted. In contrast, image analysis in the humanitarian domain is in many cases still expert and image analyst based and can only, to some extent, be supported by semi-automated tools. Typical humanitarian mappings tasks which can only partially be automated are the assessment of buildings, roads, critical infrastructure, movement of refugees/internally displaced persons (IDP) etc. This is mainly due to the fact that the features of interest are often small in size, heterogeneous and inconsistent.

The mapping scales needed for humanitarian situational awareness are demanding. Detecting the relevant objects is often difficult and challenging. Generally, image analysis requires specialized methods for specialized imagery. Very high 
spatial resolution imagery is a relatively new data type, with its own unique characteristics and derived information products. Basic methodological image analysis research in this context still has to mature.

In the following, selected relevant trends in semi-automated satellite image analysis are briefly reviewed and discussed. A focus is put on the monitoring and the analysis of refugee/IDP camps, while some of the methods may as well be used for the assessment of natural resources and land conflict situation awareness in humanitarian settings. All these methods borrow heavily from trends and research avenues in the general context of optical remote sensing image analysis, but with an additional focus on generating results in a timely manner and involving an analyst in the information extraction process.

An important step towards routine application of semiautomated image information extraction for humanitarian purposes came from the domain of Geographic Object-Based Image Analysis (GEOBIA) using image segmentation and object classification approaches [3]. Individual image objects are addressed in an iterative approach using spectral, contextual, textural as well as hierarchical information. The definition of parameters within the so-called "rule sets" is based on the experience of the image analyst. It can partially be supported by image data statistics. Such rule sets are often image/scene dependent and optimized to delineate a certain desired object/feature class in a specific type of optical satellite imagery or a given geographic setting/ environment. Transferability of rule sets can be supported by limiting the number of absolute thresholds. In case the use of absolute values is required, variables should be defined which can be adapted in a straightforward way. Robust features such as statistical object attributes which are to a large extent scene independent prove to be useful [4]. Additionally, all imagery should be corrected carefully for atmospheric, radiometric and illumination effects. Whenever such a rule set is transferred to different image categories, e.g. different spatial resolution or different spectral characteristics, some adaptation and optimization has to be carried out to achieve reliable classification.

In a very comprehensive study [5] the authors show how such GEOBIA approaches, implemented in the eCognition software, and combined with integrated spatial indicators, can be used for multi-temporal IDP camp monitoring compatible with standardized land cover classification schemes. Building on the analysis of land-use and land-cover changes in the surroundings of an IDP camp, the authors are able to describe trends in camp/population size as well as in natural resource availability and critical environmental changes around the camp. In the context of mining of conflict resources, GEOBIA methods can be applied for monitoring of changing environments around artisanal and small-scale mining (ASM) sites, even in regions that are difficult to monitor like the tropical forests [6]. This research builds on a multi-temporal and multiscale analysis approach and exploits the set-up of predeveloped sub-routines, i.e. snippets, to build an adaptable algorithm library for the analysis of potential mining areas in different conflict regions.

In order to overcome some of the limitation in the GEOBIA approaches, especially the fact that rule sets typically have to be defined in a time-consuming trail-and-error process, [7] developed a framework for fast and interactive classification of very high resolution optical imagery. The framework is based on a hierarchical Markov Random Field image modeling approach, implemented in a multi-layer quadtree structure. This allows including scale dependent image feature characteristics during the classification process. At the same time, computational efforts are being minimized. The idea for the development of this processing scheme was a very fast and interpreter based provision of training samples for an image feature class leading to a very fast, robust and reliable classification of these features in a given satellite scene. The overall workflow - including the relevant training of the algorithm as well as the automated selection of classification attributes and the computation of the full classification - should not take longer than one hour for a typical four band (RGB, NIR) 8-bit image with 10,000 by 10,000 pixels in size. Results show that the approach is fast and efficient on relatively smooth image sections (smallest quadtree element is $2 \times 2$ pixels) and does not perform so well in image regions with highly varying features within a few pixels of range only.

To address the spatial limitations caused by the simple quadtree geometry, sample supervised segment generation can be used for fast and semi-automated semantic segment generation [8]. In this approach, training samples for a relevant image feature category are provided by an analyst to train and optimize a semantic segmentation approach for the given classification problem based on automatic parameter tuning, dedicated spatial metrics and search optimization guided by metaheuristics. The primary aim for developing the image analysis approach was to allow very fast, semi-automated and robust segmentation and classification of relevant image features, based on minimum operator interaction when training the algorithm during a rapid mapping process. The approach performs fast and efficient for basic search problems and yielded good results when tested on different satellite images analyzing structures and tents/shelters of refugee camps and for mapping agricultural fields describing land use patterns.

Another interesting approach is presented by [9], where a mathematical morphology filter technique is utilized to estimate dwelling numbers in Camps in the Darfur region based on sub-meter optical satellite imagery. The technique combines multi-scale filtering and image arithmetic with parameters tuned and adapted for the specific situation of dwellings in the particular geographic region. The method is applied to different camps and results show both, under- and overestimation when compared to reference data sets generated by visual counting of dwellings and manual digitization.

\section{B. Applying Sample Supervised Segmentation to the Al Zaatari Camp in Jordan}

The sample supervised image analysis methodology [8] focusses on interactive or sample supervised processes for segmentation and classification. The method provides for both automatic and manual tuning of segmentation and classification processes. In appropriate situations a given segmentation algorithm may be automatically tailored to the elements of interest. A user digitizes only a few sample elements and an efficient search process (metaheuristic) for appropriate segmenta- 
tion algorithm parameters is carried out. In more complex scenarios an oversegmentation is advocated. The provided digitized elements are subsequently used to start a one-class classification process (e.g. one-class support vector machine). A user may interactively add new samples of the class of interest and manually tune classifier parameters and select attributes to be used in classification. Alternatively, an automatic search may also automatically tune classifier parameters and attribute subset selection, with generalizability tests implicit (active learning may also be considered in such a context). After sufficient results are achieved on such training image subsets, the stored segmentation and classification models may be run on the rest of the camp.

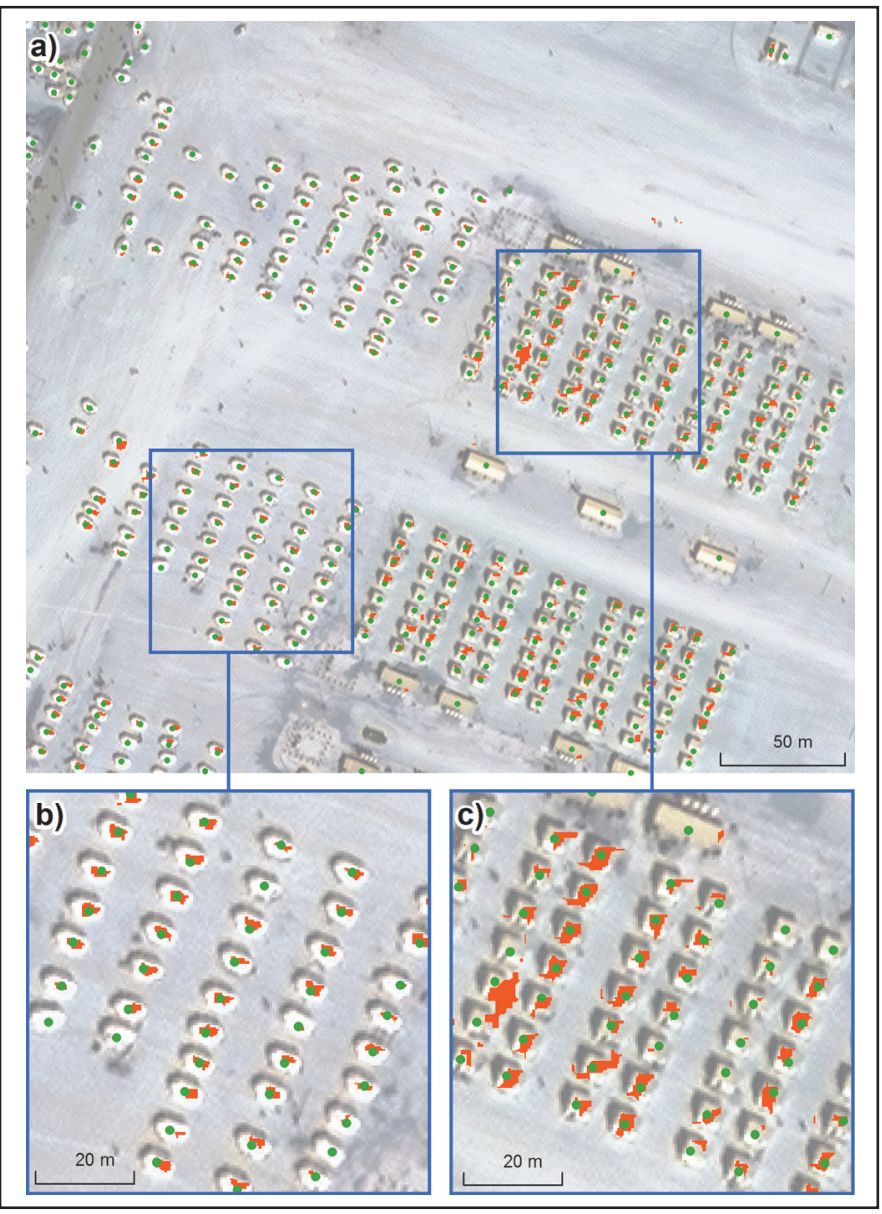

Fig. 2. Superimposition of visually/manually digitized (green dots) and semi automatically derived (red polygons) shelter locations, based on the Al Zaatari data set of WorldView-2, as of January 03, 2013. For the semi-automatic mapping a sample supervised approach was used. Part a) showing test zone of the Al Zaatri Scene. Part b) and c) showing zoom-windows with slightly different results in segmentation characteristics.

In the case of the Al Zaatari camp, a WoldView-2 Data set of January 3, 2013 provided by European Space Imaging (see Fig. 1) was used to extract shelters for a test zone in the camp, based on the sample supervised methodology [8] (see Fig. 2). Five shelter sample elements were digitized as representative of tents and structures ( $\sim 3$ minutes of operator interaction). A manually tuned over-segmentation was produced of these scenes in contrast to the automatic procedure due to the diverse element characteristics and satellite data limitations $(\sim 4$ minutes of operator interaction). For the subset, 20 additional segment samples were selected ( $\sim 2$ minutes of operator interaction). Attribute tuning and classifier free parameter tuning was performed until visually satisfactory results were obtained (3 minutes). As a refinement process and to split clustered segments/shelters, a $7 \times 7$ minimum filter kernel was applied on the result. Subsequently the built model may be run on the entire image. In the case of Al Zaatari WorldView-2 data set an estimate of 30 minutes of operator interaction and processing time would be needed. This is a significant reduction in processing and analysis time compared to the original visu$\mathrm{al} /$ manual digitalization of the shelters and tents. For the whole scene this took three analysts about $3 \mathrm{~h}$ each, resulting in a total effort of 9 hours of working time to extract the shelter/tent GIS layer for this scene.

\section{Results}

For comparison of the results of the sample supervised analysis method the resulting shelter polygons were compared to the manually/visually extracted GIS data set generated during the emergency mapping activation of the DLR/ZKI during January 2013. Figure $2 \mathrm{~b}$ shows one part of the testing zone where resulting shelter polygons were very small and partially underestimating shelter occurrence. Fig. $2 \mathrm{c}$ shows a part of the test zone, where shelter polygons resulting from the semiautomatic procedure are coarser, and partially also very faint. When comparing the geometric/thematic matches of the two data sets for the full test zone directly, 153 out of 341 visually derived centre coordinates of shelters $(44,9 \%)$ directly fell into an automatically derived shelter polygon, and mutually, 153 polygons out of $376(40,7 \%)$ directly matched geometrically with a digitized centre coordinate of a shelter. Assuming that a typical spatial distance between tents is on the order of $10 \mathrm{~m}$, a $5 \mathrm{~m}$ radius buffer was introduced for the computation of the geometrical/thematic matches. This resulted in 290 of the 341 digitized shelter locations $(85,0 \%)$ matching with an automatically derived polygon and 369 out of 376 polygons $(98,1 \%)$ matching with a shelter location. Table 1 summarises these results.

TABLE I. COMPARISON OF AUtOMATED VS. Visual/MANUAL SHELTER COUNTS

\begin{tabular}{|c|c|c|}
\hline \multicolumn{3}{|c|}{$\begin{array}{c}\text { Geometric/thematic matching between automatically and visually } \\
\text { derived shelters }\end{array}$} \\
\hline Mutual match & $\begin{array}{c}\text { Sample Supervised (total } \\
\text { polygons) matching visual } \\
\text { shelter points }\end{array}$ & $\begin{array}{l}\text { Visually derived shelter } \\
\text { points (total points) } \\
\text { matching sample su- } \\
\text { pervised polygons }\end{array}$ \\
\hline $\begin{array}{l}\text { Direct } \\
\text { geometrical } \\
\text { match }\end{array}$ & $\begin{array}{c}153(376) \\
40.7 \%\end{array}$ & $\begin{array}{c}153(341) \\
44.9 \%\end{array}$ \\
\hline $\begin{array}{l}\text { Match with when } \\
\text { using a buffer of } \\
5 \mathrm{~m} \text { distance }\end{array}$ & $\begin{array}{c}369(376) \\
98.1 \%\end{array}$ & $\begin{array}{c}290(341) \\
85.5 \%\end{array}$ \\
\hline
\end{tabular}

Table I illustrates that the sample supervised approach, although already matching the shelters quite nicely, still misses some of the shelters derived visually and still underestimates the overall number of shelters. In the example given, underestimation is by approximately $14.5 \%$. 


\section{Comparison and discussion of other methods}

The review of current developments in satellite mapping for humanitarian awareness suggests that there is a trend towards hybrid approaches: building on analytical skills of experienced image analysts, providing samples and training for respective computer-based algorithms, and then taking these samples to scale by applying the optimized rules/search schemes or parameterization of search approaches on the full satellite data sets. All five reviewed and discussed approaches (visual interpretation, object-based image analysis, quadtree, sample supervised segmentation and mathematical morphology) are still either relatively labor intensive in application (visual analysis/definition or adaptation of rule sets in GEOBIA) or have some limitations in general applicability (quadtree/sample supervised segmentation/math. morphology). Further dedicated research and development work is needed to come up with generalized and even more robust analyst and computer aided tools/frameworks to support near real time humanitarian mapping tasks. The described trend in combining visual and cognitive skills of human operators together with the systematic computational capacities of today's computing environments is expected to be the most suitable way forward in space-based humanitarian mapping. Neither visual interpretation alone, nor the attempts to fully automate the analysis tasks will yield the best, most robust and effective results.

TABLE II. COMPARISON OF APPROACHES IN SATELLITE IMAGE ANALYSIS FOR HUMANITARIAN MAPPING

\begin{tabular}{|c|c|c|c|}
\hline \multirow{2}{*}{$\begin{array}{l}\text { Satellite } \\
\text { image } \\
\text { analysis } \\
\text { ap- } \\
\text { proaches }\end{array}$} & \multicolumn{3}{|c|}{ Characteristics } \\
\hline & Key aspects & Pro & Contra \\
\hline $\begin{array}{l}\text { Visual } \\
\text { inter- } \\
\text { pretation }\end{array}$ & $\begin{array}{l}\text { Basic approach, } \\
\text { requires } \\
\text { experience, } \\
\text { involves manual } \\
\text { digitalization of } \\
\text { features }\end{array}$ & $\begin{array}{l}\text { Simple, robust, } \\
\text { cognitive skills } \\
\text { of analyst used, } \\
\text { works in ad- } \\
\text { verse imaging } \\
\text { situations }\end{array}$ & $\begin{array}{l}\text { Time-consuming, } \\
\text { maybe incon- } \\
\text { sistent if carried } \\
\text { out by multiple } \\
\text { analysts }\end{array}$ \\
\hline $\begin{array}{l}\text { Geographi } \\
\text { c Object- } \\
\text { Based } \\
\text { Image } \\
\text { Analysis }\end{array}$ & $\begin{array}{l}\text { Rule set } \\
\text { definition, } \\
\text { interactive } \\
\text { development }\end{array}$ & $\begin{array}{l}\text { Large variety of } \\
\text { options in defi- } \\
\text { nition }\end{array}$ & $\begin{array}{l}\text { Time-consuming } \\
\text { set up, limited } \\
\text { transferability, } \\
\text { complex }\end{array}$ \\
\hline Quadtree & $\begin{array}{l}\text { Simple } \\
\text { geometrical } \\
\text { hierary, } \\
\text { computational } \\
\text { effective }\end{array}$ & $\begin{array}{l}\text { Straightforward } \\
\text { in application, } \\
\text { low computa- } \\
\text { tional costs }\end{array}$ & $\begin{array}{l}\text { Blocking effect in } \\
\text { scale analysis, } \\
\text { works best on } \\
\text { relatively homo- } \\
\text { geneous image } \\
\text { regions }\end{array}$ \\
\hline $\begin{array}{l}\text { Sample } \\
\text { supervised } \\
\text { segment- } \\
\text { ation }\end{array}$ & $\begin{array}{l}\text { Automated } \\
\text { semantic } \\
\text { segment } \\
\text { generation }\end{array}$ & $\begin{array}{l}\text { Segmentation } \\
\text { optimized for } \\
\text { training samples }\end{array}$ & $\begin{array}{l}\text { Computationally } \\
\text { expensive, may be } \\
\text { limited in com- } \\
\text { plex imaging } \\
\text { conditions }\end{array}$ \\
\hline $\begin{array}{l}\text { Mathe- } \\
\text { matical } \\
\text { morph- } \\
\text { ology }\end{array}$ & $\begin{array}{l}\text { Multi-scale } \\
\text { filtering and } \\
\text { image } \\
\text { arithmetic } \\
\text { operations }\end{array}$ & $\begin{array}{l}\text { Simple and } \\
\text { robust computa- } \\
\text { tions }\end{array}$ & $\begin{array}{l}\text { Parameters fixed } \\
\text { for a given geo- } \\
\text { graphic setting, } \\
\text { over and under- } \\
\text { estimation arti- } \\
\text { facts }\end{array}$ \\
\hline
\end{tabular}

The best synthesis of the different approaches will combine interpreter/analyst and computer aided feature sample selection to train semantically optimized segmentation of imagery together with automated selection of most discriminative object attributes for highly robust and reliable object/feature segmentation and classification.

\section{CONCLUSION}

Building on the findings of the discussed research work it is recommended to invest more development work into interactive computer aided and semi-automated methods for assessing and monitoring of humanitarian situations by means of very high resolution optical imagery. For fast and reliable mapping of camps, natural resources or general spatial patterns related to humanitarian situations, future analysis and mapping schemes should have well shaped capabilities for interaction with human analyst and image interpretation experts to identify/mark relevant features such as tents/shelters or relevant geospatial changes in respective imagery. Secondly, algorithms and capable search strategies should be used to apply the interactively identified and most discriminative features on large satellite data sets during the rapid mapping and analysis processes for humanitarian mapping tasks. Finally, it is envisaged that automation in the humanitarian rapid mapping domain will increase alongside the general improvement of methods in optical high spatial resolution remote sensing.

\section{REFERENCES}

[1] S. Voigt, T. Kemper, T. Riedlinger, R. Kiefl, K. Scholte, and H. Mehl, „Satellite Image Analysis for Disaster and Crisis-Management Support," IEEE Transactions on Geosience and Remote Sensing, 45(6), 2007, pp. 1520-1528.

[2] D. Tiede, P. Füreder, S. Lang, D. Hölbling, and P. Zeil, „Automated Analysis of Satellite Imagery to provide Information Products for Humanitarian Relief Operations in Refugee Camps from Scientific Development towards Operational Services," PhotogrammetrieFernerkundung-Geoinformation, 3, 2013, pp. 185-195.

[3] E. Schöpfer, S. Lang, and J. Strobl, „Segmentation and object-based image analysis," in T. Rashed, and C. Jürgens, eds., Remote sensing of urban and suburban areas. Berlin, Springer, 2010, pp. 181-192.

[4] D. Tiede, S. Lang, D. Hölbling, and P. Füreder, „Transferability of OBIA rulesets for IDP Camp Analysis in Darfur," in E. A. Addink, and F.M.B.V. Coillie, eds., ISPRS Vol.No. XXXVIII-4/C7, 2010.

[5] M. Hagenlocher, S. Lang, and D. Tiede, „Integrated assessment of the environmental impact of an IDP camp in Sudan based on very high resolution multi-temporal satellite imagery," Remote Sensing of Environment, 126, 2012, pp. 27-38.

[6] E. Schoepfer, and O. Kranz, „Monitoring natural resources in conflict using an object-based multiscale image analysis approach," in E. A. Addink, and F.M.B.V. Coillie, eds., ISPRS Vol.No. XXXVIII-4/C7, 2010 .

[7] J. Kersten, M. Gähler, and S. Voigt, „A general framework for fast and interactive classification of optical vhr satellite imagery using hierarchical and planar Markov random fields," PhotogrammetrieFernerkundung-Geoinformation, 6, 2010, pp. 439-449.

[8] C. Fourie, and E. Schoepfer, "Data Transformation Functions for Expanded Search Spaces in Geographic Sample Supervised Segment Generation," Remote Sens., 6(5), 2014, pp. 3791-3821.

[9] T. Kemper, M. Jenerowicz, P. Soille, and M. Pesaresi, "Enumeration of dwellings in Darfur Camps from GeoEye-1 satellite images using mathematical morphology", IEEE Journal of Selected Topics in Applied Earth Observations and Remote Sensing, 2011, vol. 4, pp. 8-15. 